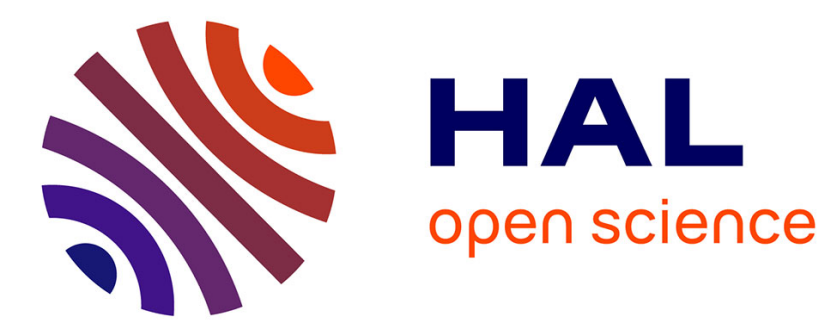

\title{
INPUT IMPEDANCE OF PLANAR FP AND EBG ANTENNAS
}

Thai Hung Vu, Kouroch Mahdjoubi, Sylvain Collardey, Anne-Claude Tarot

\section{To cite this version:}

Thai Hung Vu, Kouroch Mahdjoubi, Sylvain Collardey, Anne-Claude Tarot. INPUT IMPEDANCE OF PLANAR FP AND EBG ANTENNAS. PID, Apr 2007, France. hal-00142200

\section{HAL Id: hal-00142200 \\ https://hal.science/hal-00142200}

Submitted on 17 Apr 2007

HAL is a multi-disciplinary open access archive for the deposit and dissemination of scientific research documents, whether they are published or not. The documents may come from teaching and research institutions in France or abroad, or from public or private research centers.
L'archive ouverte pluridisciplinaire HAL, est destinée au dépôt et à la diffusion de documents scientifiques de niveau recherche, publiés ou non, émanant des établissements d'enseignement et de recherche français ou étrangers, des laboratoires publics ou privés. 


\title{
INPUT IMPEDANCE OF PLANAR FP AND EBG ANTENNAS
}

\author{
Thai-Hung VU, Kourosh MAHDJOUBI, Sylvain COLLARDEY, Anne-Claude TAROT \\ IETR, UMR CNRS 6164, Université de Rennes 1, Beaulieu Campus, 35042 Rennes Cedex, \\ France \\ Email: thai-hung.vu@univ-rennes1.fr ; mahdjoubi@univ-rennes1.fr
}

\begin{abstract}
The theoretical study of the input impedance for Fabry-Perot (FP), Electromagnetic Band Gap (EBG) or Leaky Wave (LW) antennas is a real challenge from mathematical and electromagnetic modelling point of view and is very rarely addressed in the literature. In this paper, an original method will be presented to evaluate the input impedance of the EBG antennas excited by a line source or a point source. The method is based on the generalisation of the plane wave expansion of the antenna internal fields to cylindrical or spherical wave expansions. This procedure leads to simple and rigorous formulas which are very helpful for designing the EBG, FP and LW antennas and for understanding their electromagnetic behaviours.
\end{abstract}

\section{Introduction}

EBG antennas are generally composed of a (multilayer) EBG material (Electromagnetic Band Gap), a reflector plane and a primary source that is placed inside the structure (Fig. 1). These recent radiating structures are nowa-days applied to produce highly directive beams. Compared to classical directive antennas (horn, parabolic reflector, etc) EBG antennas are very compact and low profile, but have a very narrow bandwidth.

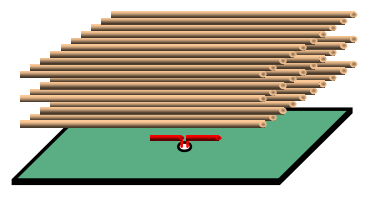

Fig. 1.Typical structure of an EBG antenna

The mathematical investigation on antennas input impedance has always been a delicate task due to the sigularity of near field in the vicinity of the exciting source. However, the antenna far field (radiation pattern) and the general properties of EBG structures (dispersion diagram, transmission and reflection coefficients ...) can be studied relatively easily by the plane wave excitation method or by the equivalent transmission line method.

In this communication, we will apply as a first stage, the plane wave method to the internal field of Fabry-Perot (FP) antennas to extract the expression of the input impedance of ideal plane wave sources. The procedure is then generalised for more realistic excitation sources. Hence, we will propose new methods that allow obtaining the input impedance of FP or EBG antennas excited by a line source or a point source. The method leads to simple and rigorous analytical formulas which are helpful for the design of EBG antennas and gives a better comprehension of the physical phenomena.

\section{Plane Wave Input Impedance of Fabry-Perot (FP) Antennas}

Let us consider a TM plane wave source inside a Fabry Perot cavity made of two infinite Partially Reflective Surfaces (PRS) characterized by their transmission $\left(t_{1} ; t_{2}\right)$ and reflexion coefficients $\left(r_{1} ; r_{2}\right)$ (Fig. 2). By applying the method of the successive reflections inside the cavity, the external (transmitted) and internal fields can be written as a superposition of all partially transmitted or reflected fields [1, 2].

$$
\begin{aligned}
& T_{F P}(\theta)=\frac{E_{z}^{\text {trans }}}{E_{z}^{\text {inc }}}=t_{1}\left(1+\sum_{n=1}^{\infty}\left(r_{1} r_{2}\right)^{n} \exp (-2 j k n D \cos (\theta))+\sum_{n=1}^{\infty} r_{2}\left(r_{1} r_{2}\right)^{n} \exp \left(-2 j k n D \cos (\theta)-2 j k D_{2} \cos (\theta)\right)\right)=\frac{t_{1}\left(1+r_{2} \exp \left(-2 j k D_{2} \cos (\theta)\right)\right)}{1-r_{1} r_{2} \exp (-2 j k D \cos (\theta))} \\
& E^{\text {int }}=E_{z}^{\text {inc }}\left(1+\sum_{n=1}^{\infty}\left(r_{1} r_{2}\right)^{n} \exp (-2 j k n D \cos (\theta))+\sum_{n=1}^{\infty} r_{2}\left(r_{1} r_{2}\right)^{n} \exp \left(-2 j k n D \cos (\theta)-2 j k D_{2} \cos (\theta)\right)\right)=\frac{\left(1+r_{2} \exp \left(-2 j k D_{2} \cos \theta\right)\right)}{1-r_{1} r_{2} \exp (-2 j k D \cos \theta)} E_{z}^{\text {inc }} \\
& H_{x}^{\mathrm{int}}=\frac{\sin \theta}{1-r_{1} r_{2} \exp (-2 j k D \cos \theta)} H^{\text {inc }} \quad H_{Y}^{\mathrm{int}}=\frac{\cos \theta\left(1+r_{2} \exp \left(-2 j k D_{2} \cos \theta\right)\right)}{1-r_{1} r_{2} \exp (-2 j k D \cos \theta)} H^{i n c}
\end{aligned}
$$


The magnetic field $\mathrm{H}$ has naturaly two components $\mathrm{Hx}$ and Hy. The resulting $\mathrm{Hx}$ and Hy given in relation (3) lead to two distinct impedance expressions called here $Z_{\text {guide }}$ and $Z_{\text {standing }}$ corresponding to the guided and standing waves inside the FP cavity (see Fig.2).

$$
Z_{\text {guided }}(\theta)=\frac{E_{z}^{\text {int }}}{H_{x}^{\text {int }}}=\frac{\eta}{\sin \theta} \quad Z_{s \tan \operatorname{ding}}(\theta)=\frac{E_{z}^{\text {int }}}{H_{y}^{\text {int }}}=\eta \frac{\left(1+r_{2} \exp (-2 j k D 2 \cos \theta)\right)}{\cos \theta\left(1-r_{2} \exp (-2 j k D 2 \cos \theta)\right)}
$$

$\eta$ is the intrinsic impedance of plane wave in free space.

If the ideal plane wave source is replaced by a real excitation source of finite size (Fig. 1a), the radiation pattern $\mathrm{P}_{\mathrm{FP}}(\theta)$ of the new FP antenna can be obtained by the multiplication of the excitation source pattern $\mathrm{P}_{\text {source }}(\theta)$ and the FP response $T_{F P}(\theta)$ (Eq. 1): $P_{F P}(\theta)=P_{\text {source }}(\theta) T_{F P}(\theta)$. The reason is that $P_{\text {source }}(\theta)$ is resulted in fact from the plane wave expansion of the source far field. To obtain the input impedance of the new FP antenna, one can apply a similar method by using the plane wave expansion of real source near field. However, three major difficulties appear in this case. First, the mathematical singularity of the near field (due to the stored energies) requires a very large number of evanescent and complex plane wave terms. Second, the transmission and reflection coefficients $\left(t_{1} ; t_{2}\right)$ and $\left(r_{1} ; r_{2}\right)$ should be evaluated for all these evanescent plan waves. This is not a simple task. Third, the real source has a unique value for its input impedance, but for plane wave expansions, we have at least two different expressions (Eq. 4). We should therefore recombine properly the near field expressions to obtain a unique impedance value.

We should add that all the commercial electromagnetic solvers produce easly the radiation pattern for any arbitrary excitation source, but none of them give the plane wave expansion of the near field. We conclude that the plane wave expansion method is not a practical way to obtaine the input impedance of the FP and EBG antennas excited by realistic and finite size sources. In the following sectoins we wil apply the cylindrical and spherical expansions to extract relatively easily the imput impedance of line and point sources respectively. Although these are still ideal sources, they seem to be more realistic sources for EBG antennas.
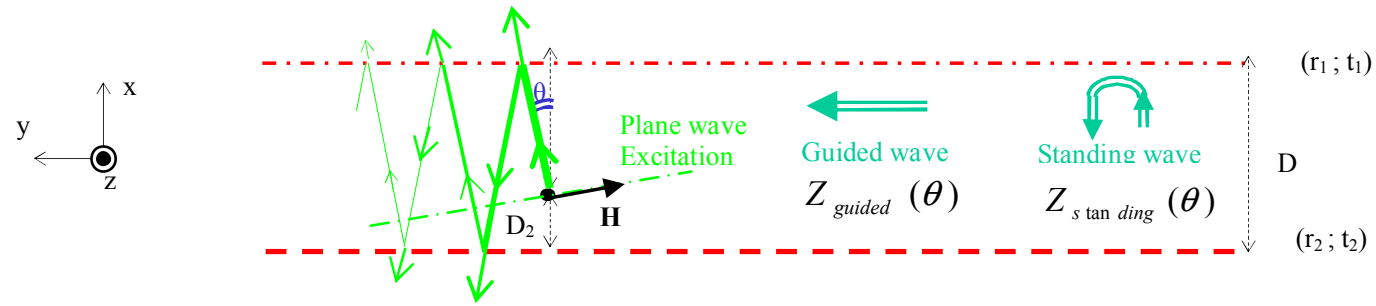

Fig. 2. Guided \& standing wave inside the cavity

\section{Cylindrical Wave Response and Input Impedance}

Let us consider a line source (infinitely long current source) placed in front of a PRS. In figure 3, an FDTD simulation shows the interaction between the cylindrical wave (created by the line source) and the PRS. The incident cylindrical wave is obviously centred on the exciting line source. After having met the PRS, two new waves appear: the "transmitted" wave, which remains centred on the primary source, and the "reflected" wave, which is centred on the image of the excitation source through the PRS. We should notice that the two waves are always cylindrical ones.

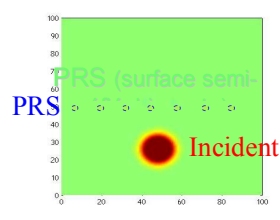

Incident cylindrical wave $\mathrm{H}_{0}{ }^{(2)}$

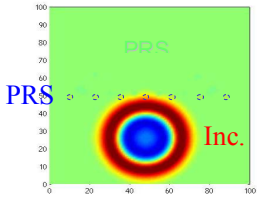

Incident cylindrical wave $\mathrm{H}_{0}{ }^{(2)}$

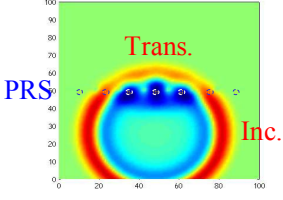

Incident cylindrical wave arrives on the PRS

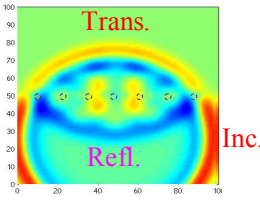

Reflected \& transmitted cvlindrical wave

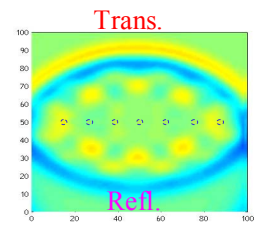

Reflected \& transmitted cvlindrical wave

Fig. 3. Interaction between a cylindrical wave and a Partially Refflective Surface (PRS ) 
Let us now consider the FP cavity excited with a line source (Fig. 4). By using the method of successive reflexions applied in the first part for the plane waves and illustrated in Fig. 4 for cylindrical waves, we obtain the analytical expression of the electric field $\mathrm{E}$ and the magnetic field $\mathrm{H}$ inside the cavity:

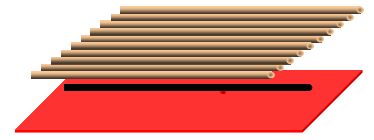

Fig. 4. Fabry-Perot cavity excited by a line source

$$
\begin{aligned}
& E=k^{2}\left(H_{0}^{(2)}\left(0^{+}\right)+r_{c 1} \sum_{n=0}^{\infty}\left(r_{c 1} r_{c 2}\right)^{n} H_{0}^{(2)}\left(2 k\left(D_{1}+n D\right)\right)+r_{c 2} \sum_{n=0}^{\infty}\left(r_{c 1} r_{c 2}\right)^{n} H_{0}^{(2)}\left(2 k\left(D_{2}+n D\right)\right)+2 \sum_{n=1}^{\infty}\left(r_{c 1} r_{c 2}\right)^{n} H_{0}^{(2)}(2 k n D)\right) \\
& H=k^{2}\left(\frac{H_{1}^{(2)}\left(0^{+}\right)}{j \eta}\right)
\end{aligned}
$$

Where $r_{c 1}$ and $r_{c 2}$ are the reflection coefficient of the PRS of the FP cavity for cylindrical waves, $\mathrm{H}_{0}{ }^{(2)}, \mathrm{H}_{1}{ }^{(2)}$ are Hankel functions of order zero and 1 consecutively and of second kind (output waves) [3].

The input impedance of the exciting (line) source becomes simply the ratio of electrical field and magnetic field at the source point $\left(D_{1}\right)$ :

$$
Z=\frac{E}{H}=Z_{\text {source }}+\frac{j \eta}{H_{1}^{(2)}\left(0^{+}\right)}\left(r_{c 1} \sum_{n=0}^{\infty}\left(r_{c 1} r_{c 2}\right)^{n} H_{0}^{(2)}\left(2 k\left(D_{1}+n D\right)\right)+r_{2} \sum_{n=0}^{\infty}\left(r_{1} r_{2}\right)^{n} H_{0}^{(2)}\left(2 k\left(D_{2}+n D\right)\right)+2 \sum_{n=1}^{\infty}\left(r_{c 1} r_{c 2}\right)^{n} H_{0}^{(2)}(2 k n D)\right)
$$

Figure (6) shows that there is a very good agreement between the relation (7) and the input impedance calculated by the FDTD method.

It should be noted that our results can be applied to multilayer EBG structures by using a simple recursive method developed in [1]

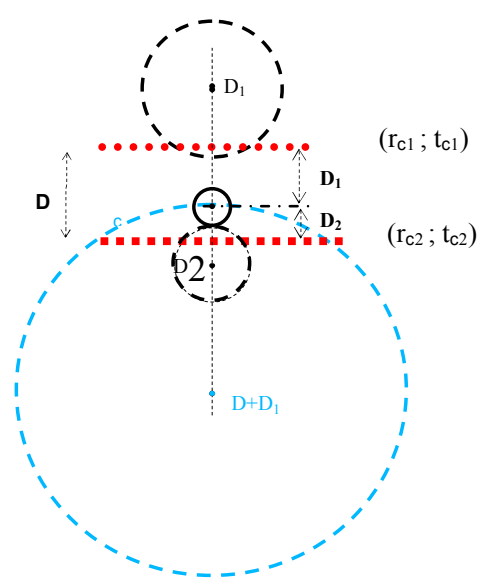

Fig.5. Images successive of a cylindrical wave source are also cylindrical wave sources

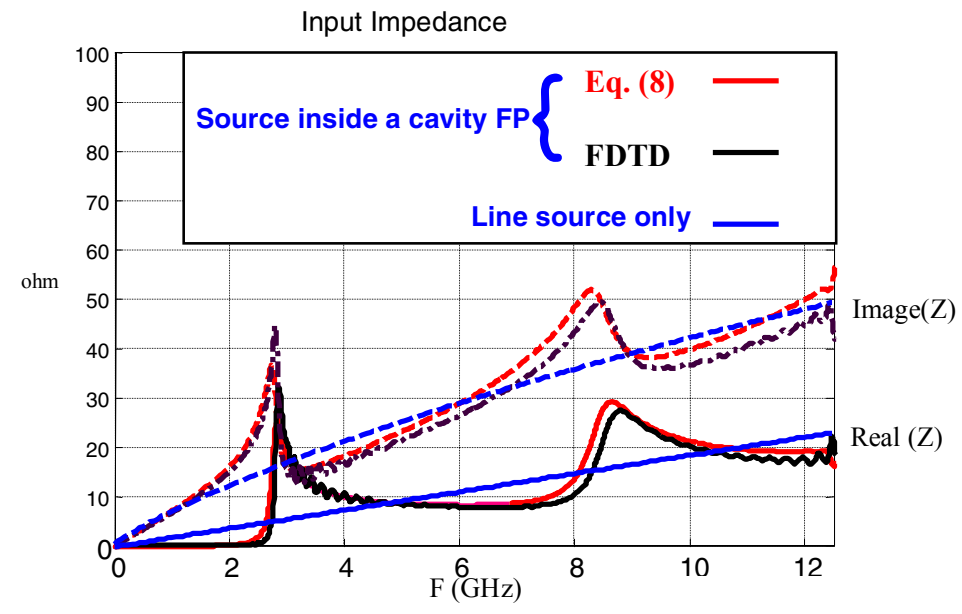

Fig.6. Input impedance of a infinitely long current source placed inside a cavity

\section{Input Impedance of FP Antennas Excited by Point Source or Short}

Fig. 7 shows a symmetrical FP cavity excited with a point source or short dipole antenna. The electric and magnetic field of the dipole alone are classically given by the following expressions [4]:

$$
\begin{aligned}
& H_{\phi}=\frac{I l}{4 \pi r^{2}}(1+j k r) e^{-j k r} \sin \theta \\
& E_{r}=-j \frac{\eta}{k} \frac{I l}{2 \pi r^{3}}(1+j k r) e^{-j k r} \cos \theta \\
& E_{\theta}=-j \frac{\eta}{k} \frac{I l}{4 \pi r^{3}}\left(1+j k r-k^{2} r^{2}\right) e^{-j k r} \sin \theta
\end{aligned}
$$

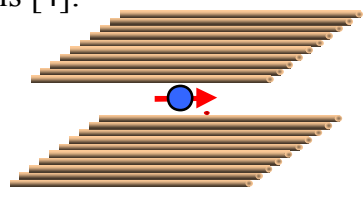

Fig. 7. Symmetrical Fabry-Perot cavity excited by a short dipole

where, $I$ and $l$ are respectively the dipole current and length. An approximate expression of the complex input impedance can be obtained by using the Pointing theorem to evaluate the dipole complex power P:

$$
P=\frac{1}{2} I^{2} Z=\frac{1}{2} \oiint_{S}\left(\vec{E}_{\theta} \wedge \vec{H}_{\phi}^{*}\right) d \vec{s} \quad \Rightarrow \quad Z_{\text {in }}^{\text {dipole }}=K \frac{\eta}{6 \pi} \frac{\left(1-j k r_{0}\right)\left(1+j k r_{0}-k^{2} r_{0}^{2}\right)}{j k r_{0}^{3}}
$$


$r_{0}$ is the radius of the sphere surface $S$ for the integral evaluation. Obviously, in these expressions, the stored energies inside the sphere are neglected. The imaginary part of the input impedance is therefore not exact. We have introduced a factor $\mathrm{K}$ and adjusted empirically the parameters $\mathrm{r}_{0}$ and $\mathrm{K}$ to obtain a good agreement with the numerical results obtained by FDTD method (Fig. 8).
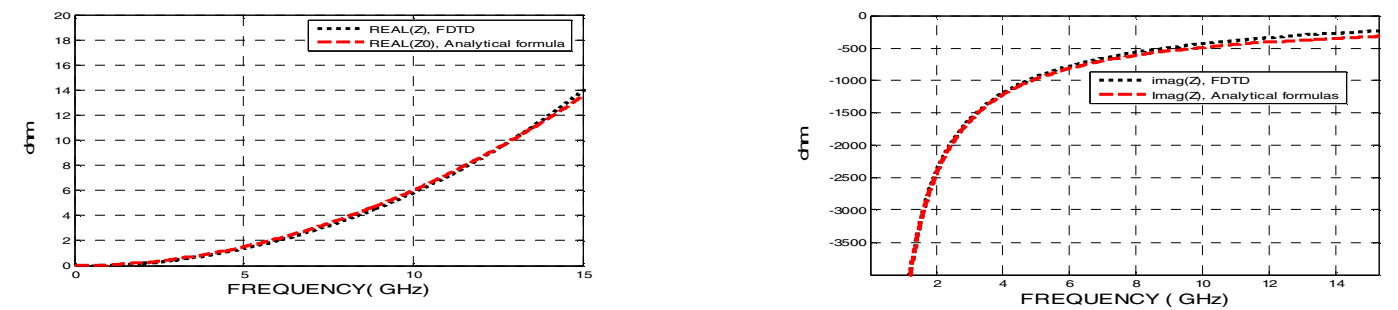

Fig. 8. Real (left) and imaginary (right) parts of the an isolated short dipole of $\mathrm{l}=5 \mathrm{~mm}$, obtained by FDTD method and by the approximate equation (12)

By applying now the same successive reflexions and image method as in the previous section (Fig. 5), we obtain the analytical expression of the input impedance of the short dipole placed in the middle of the symmetrical FP cavity (Fig. 7):

$Z=K \frac{\eta}{6 \pi} \frac{\left(1-j k r_{0}\right)}{j k} e^{j k r_{0}}\left[E_{k r}\left(r_{0}\right)+r_{1} \sum_{n=0}^{\infty}\left(r_{1} r_{2}\right)^{n} E_{k r}\left(2 D_{1}+2 n D\right)+r_{2} \sum_{n=0}^{\infty}\left(r_{1} r_{2}\right)^{n} E_{k r}\left(2 D_{2}+2 n D\right)+2 \sum_{n=1}^{\infty}\left(r_{1} r_{2}\right)^{n} E_{k r}(2 n D)\right]$

Where $_{E_{k r}}=\frac{\left[1+j k r+(j k r)^{2}\right]}{r^{3}} \exp (-j k r)$ and $\mathrm{r}_{1}$ and $\mathrm{r}_{2}$ are the PRS reflection coefficients evaluated numerically

(FDTD method) for short dipole excitation. The input impedance of the resulting structure obtained from the analytical expression (12) shows a good and encouraging agreement with FDTD simulations (Fig. 9).
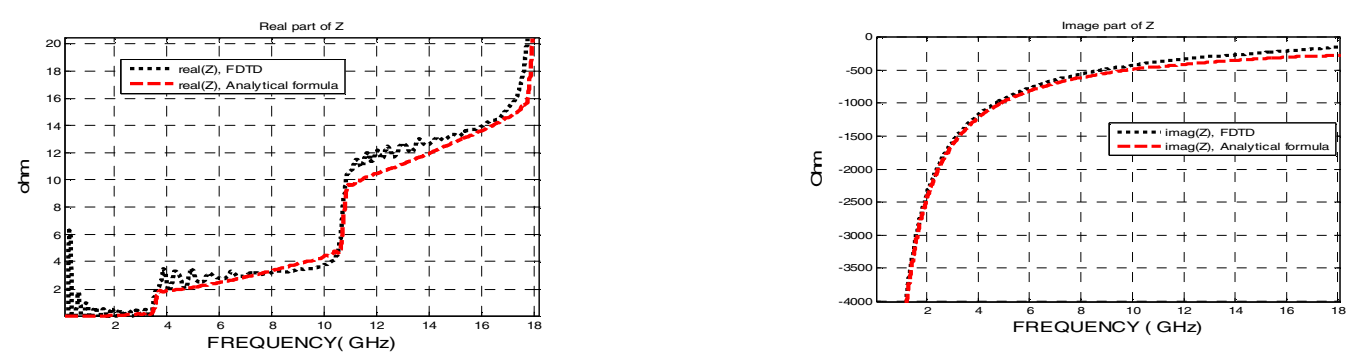

Fig. 9. Impedance of a short dipole $(l=5 \mathrm{~mm})$ inside a symmetrical FP cavity of $D=40 \mathrm{~mm}(\mathrm{D1}=\mathrm{D2})$. , surfaces of metallic wires with $\mathrm{a} / \mathrm{Pt}=5 \%, \mathrm{Pt}=3 \mathrm{~mm}$, diameter of wires, $\mathrm{Pt}$ : distance between wires

\section{Discussion \& Conclusion}

We presented an original theory to predict the input impedance of FP antennas from the knowledge of the near filed of the exciting source alone. Based on the image principle, the elaborated analytical expressions are fast and simple and produce reliable results with good precision for antenna design. Through a recursive procedure elaborated in [1], this method can be applied to planar EBG antennas which are similar to FP ones, but have multilayer PRS walls.

In the method presented here, one requires to evaluate the reflection coefficient of the PRS walls for the exciting source imbedded in the EBG or FP structure. This is not a major issue and the most of the commercial electromagnetic softwares and the known numerical methods allow to obtain these data. In this work we applied the FDTD method to evaluate the PRS reflection and transmission coefficients for plane wave excitation $\left(\mathrm{r}_{1}, \mathrm{t}_{1}, \mathrm{r}_{2}\right.$ in Eq. 1-4), cylindrical excitation $\left(r_{c 1}, r_{c 2}\right.$ in Eq. 7) and source point excitation $\left(r_{1}, r_{2}\right.$ in Eq. 12). The method remains general and applicable to arbitrary sources. We plan to employ it soon for other kinds of primary sources such as slot or patch antennas.

\section{References}

[1] H. Boutayeb, K. Mahdjoubi and A.C Tarot, "Multi-layer crystals of metallic wires: Analysis of the transmission coefficient for outside and inside excitation", Progress In Electromagnetics Research, PIER, 59, pp. 299-324, 2006.

[2] T.H. VU, Master thesis, IETR, University of Rennes I, France, June 2005.

[3] Julius Adam Stratton, Electromagnetic theory, vol. 2. McGraw-Hill Book Company, pp. 355-364, 1941.

[4] C. Balanis, Antenna Theory, Analysis \& Design. Harper \& Row Publisher, New York, 1982 